Supporting Informations for the Communication

Entitled

\title{
A Stable Schrock-type Hafnium-Silylene Complex
}

Norio Nakata, Toshiyuki Fujita, and Akira Sekiguchi*

Department of Chemistry, Graduate School of Pure and Applied Sciences,

University of Tsukuba, Tsukuba, Ibaraki 305-8571, Japan 


\section{Experimental Section}

General Procedure. All experiments were performed using high-vacuum line techniques or in an argon atmosphere of MBRAUN MB 150B-G glove-box. All solvents were predried over sodium benzophenone ketyl and finally dried and degassed over potassium mirror in vacuum prior to use. NMR spectra were recorded on a Bruker AC-300FT NMR spectrometer ( ${ }^{1} \mathrm{H}$ NMR at $300.13 \mathrm{MHz}$; ${ }^{13} \mathrm{C}$ NMR at $75.47 \mathrm{MHz} ;{ }^{29} \mathrm{Si} \mathrm{NMR}$ at $59.63 \mathrm{MHz} ;{ }^{31} \mathrm{P} \mathrm{NMR}$ at $121.50 \mathrm{MHz}$ ). UV-spectra were recorded on a Shimadzu UV-3150 UV-Vis spectrophotometer in hexane.

Generation of 16-Electron Hafnium-Silylene Complex, $\left(\eta-\mathrm{C}_{5} \mathrm{H}_{4} \mathrm{Et}\right)_{2} \mathrm{Hf}=\mathrm{Si}\left(\mathrm{SiMe}^{t} \mathrm{Bu}_{2}\right)_{2} \quad(2)$. Bis(di-tert-butylmethylsilyl)dilithiosilane 1[1], prepared from 1,1-bis(di-tert-butylmethylsilyl)-2,3bis(trimethylsilyl)-1-silacycloprop-2-ene $(53 \mathrm{mg}, 0.099 \mathrm{mmol})$ and lithium $(15 \mathrm{mg}, 2.20 \mathrm{mmol})$ in THF at room temperature, was reacted with 0.9 equiv of $\left(\eta-\mathrm{C}_{5} \mathrm{H}_{4} \mathrm{Et}\right)_{2} \mathrm{HfCl}_{2}(30 \mathrm{mg}, 0.069 \mathrm{mmol})$ in dry toluene $(1.0 \mathrm{~mL})$ for $30 \mathrm{~min}$ at $-50^{\circ} \mathrm{C}$ to form a dark green solution. After removal of inorganic salts, the solvent was evaporated at low temperature. NMR spectra at $243 \mathrm{~K}$ in toluene- $\mathrm{d}_{8}$ showed resonance signals due to bis(di-tert-butylmethylsilyl)silanediylbis(ethylcyclopentadienyl) hafnium(IV) (2) as the main product, but this complex could not be isolated in a pure form due to its thermal instability. ${ }^{1} \mathrm{H}$ NMR $\left(243 \mathrm{~K}\right.$, toluene- $\left.\mathrm{d}_{8}, \delta\right) 0.82$ (br s, $\left.3 \mathrm{H}\right), 0.93$ (br s, $\left.3 \mathrm{H}\right), 1.09(\mathrm{~m}, 6 \mathrm{H})$, 1.24 (br s, $27 \mathrm{H}), 1.43$ (br s, 9 H), 2.30 (m, 4 H), 4.77 (br s, $1 \mathrm{H}), 5.10$ (br s, $1 \mathrm{H}), 5.45$ (br s, $1 \mathrm{H}$ ), 5.84 (br s, $1 \mathrm{H}), 5.90$ (br s, $1 \mathrm{H}), 5.99$ (br s, $1 \mathrm{H}), 7.25$ (br s, $1 \mathrm{H}), 7.39$ (br s, $1 \mathrm{H}) ;{ }^{13} \mathrm{C}$ NMR $(243 \mathrm{~K}$, toluene- $\mathrm{d}_{8}$, \&) $2.1\left(\mathrm{SiCH}_{3}\right), 4.9\left(\mathrm{SiCH}_{3}\right), 16.5\left(\mathrm{CH}_{2} \mathrm{CH}_{3}\right), 18.0\left(\mathrm{CH}_{2} \mathrm{CH}_{3}\right), 23.4\left(\mathrm{CCH}_{3}\right), 23.7\left(\mathrm{CCH}_{3}\right)$, $24.0\left(\mathrm{CH}_{2} \mathrm{CH}_{3}\right), 24.2\left(\mathrm{CH}_{2} \mathrm{CH}_{3}\right), 25.5\left(\mathrm{CCH}_{3}\right), 26.2\left(\mathrm{CCH}_{3}\right), 31.5\left(\mathrm{CCH}_{3}\right), 32.2\left(\mathrm{CCH}_{3}\right), 32.9\left(\mathrm{CCH}_{3}\right)$, $33.0\left(\mathrm{CCH}_{3}\right), 102.2(\mathrm{Cp}), 103.0(\mathrm{Cp}), 104.2(\mathrm{Cp}), 105.7(\mathrm{Cp}), 108.2(\mathrm{Cp}), 109.4(\mathrm{Cp}), 112.0(\mathrm{Cp})$, 112.6 (Cp), $119.7\left(2 \mathrm{Cp}_{\text {ipso }}\right) ;{ }^{29} \mathrm{Si}$ NMR $\left(243 \mathrm{~K}\right.$, toluene-d $\left.\mathrm{d}_{8}, \delta\right) 18.3\left(\mathrm{SiMe}^{t} \mathrm{Bu}_{2}\right), 23.4\left(\mathrm{SiMe}^{t} \mathrm{Bu}_{2}\right), 123.5$ 
$(\mathrm{Si}=\mathrm{Hf}) ; \mathrm{UV} / \mathrm{Vis}$ (toluene, $223 \mathrm{~K}) \lambda_{\max } / \mathrm{nm} 411,635$.

Thermal Stability of 2. A solution of $2(0.069 \mathrm{mmol})$ in dry toluene $(1.0 \mathrm{~mL})$ was allowed to warm from $-50{ }^{\circ} \mathrm{C}$ to $0{ }^{\circ} \mathrm{C}$, the dark green color of 2 gradually changed to yellow within 10 min to form the intramolecular C-H insertion metallacycle, bis(ethylcyclopentadienyl)-2-(di-tert-butylmethylsilyl)-3tert-butyl-3,4,4-trimethyl-2,3-disilahafnacyclopentane (3) in $>80 \%$ yield by ${ }^{1} \mathrm{H}$ NMR spectrum. ${ }^{1} \mathrm{H}$ $\operatorname{NMR}\left(400.0 \mathrm{MHz}, 298 \mathrm{~K}, \mathrm{C}_{6} \mathrm{D}_{6}, \delta\right)-0.26\left(\mathrm{~d}, J_{\mathrm{CH}}=13.3 \mathrm{~Hz}, 1 \mathrm{H}\right), 0.32(\mathrm{~s}, 3 \mathrm{H}), 0.43(\mathrm{~s}, 3 \mathrm{H}), 0.61(\mathrm{~d}$, $\left.J_{\mathrm{CH}}=13.3 \mathrm{~Hz}, 1 \mathrm{H}\right), 0.96\left(\mathrm{t}, J_{\mathrm{CH}}=7.4 \mathrm{~Hz}, 3 \mathrm{H}\right), 0.98\left(\mathrm{t}, J_{\mathrm{CH}}=7.4 \mathrm{~Hz}, 3 \mathrm{H}\right), 1.17(\mathrm{~s}, 9 \mathrm{H}), 1.25(\mathrm{~s}, 9$ H), $1.30(\mathrm{~s}, 9 \mathrm{H}), 1.31$ (s, $3 \mathrm{H}), 1.32(\mathrm{~s}, 3 \mathrm{H}), 1.86-2.01(\mathrm{~m}, 4 \mathrm{H}), 3.08$ (s, $1 \mathrm{H}, \mathrm{SiH}), 5.15$ (br s, $2 \mathrm{H})$, 5.44 (br s, $2 \mathrm{H}), 6.55$ (br s, $1 \mathrm{H}), 6.78$ (br s, $1 \mathrm{H}), 7.12$ (br s, $1 \mathrm{H}), 7.60$ (br s, $1 \mathrm{H}) ;{ }^{13} \mathrm{C}$ NMR $(298 \mathrm{~K}$, $\left.\mathrm{C}_{6} \mathrm{D}_{6}, \delta\right)-2.0\left(\mathrm{SiMe} \mathrm{Bu}_{2}\right), 2.2$ (ring-SiCH $\left.\mathrm{H}_{3}\right), 16.0\left(\mathrm{CH}_{2} \mathrm{CH}_{3}\right), 16.2\left(\mathrm{CH}_{2} \mathrm{CH}_{3}\right), 21.6$ (ring-SiCCH$)$, $22.1\left(\mathrm{SiCCH}_{3}\right), 22.2\left(\mathrm{CH}_{2} \mathrm{CH}_{3}\right), 23.4\left(\mathrm{CH}_{2} \mathrm{CH}_{3}\right), 30.4\left(\mathrm{CCH}_{3}\right), 30.6\left(\mathrm{CCH}_{3}\right), 31.4$ (ring-SiC $\left.\left(\mathrm{CH}_{3}\right)_{2}\right)$, 35.2 (ring $-\mathrm{CCH}_{3}$ ), 36.1 (ring- $\mathrm{CCH}_{3}$ ), 38.1 (ring- $\mathrm{CCH}_{3}$ ), $75.2\left(\right.$ ring $\left.-\mathrm{CH}_{2}\right), 105.1(\mathrm{Cp}), 107.1(\mathrm{Cp})$, 107.6 (Cp), $108.7(\mathrm{Cp}), 109.3(\mathrm{Cp}), 112.7(\mathrm{Cp}), 114.3(\mathrm{Cp}), 114.6(\mathrm{Cp}), 128.7\left(\mathrm{Cp}_{\mathrm{ipso}}\right), 129.8\left(\mathrm{Cp}_{\mathrm{ipso}}\right)$; ${ }^{29} \mathrm{Si}$ NMR $\left(298 \mathrm{~K}, \mathrm{C}_{6} \mathrm{D}_{6}, \delta\right)-51.7(\mathrm{Hf}-\mathrm{SiH}), 24.4\left(\mathrm{SiMe}^{t} \mathrm{Bu}_{2}\right), 33.6(\mathrm{Hf}-\mathrm{Si}-\mathrm{Si})$.

Reactions of 2 with $\mathrm{MeOH}$ and $\mathrm{D}_{\mathbf{2}} \mathbf{O}$. $\mathrm{MeOH}(0.1 \mathrm{~mL})$ was added to a solution of $2(0.069 \mathrm{mmol})$ in dry toluene $(1.0 \mathrm{~mL})$ at $-50{ }^{\circ} \mathrm{C}$. Stirring of the mixture at room temperature for $10 \mathrm{~min}$ immediately resulted in the disappearance of the dark green color due to 2 . The solvent was then evaporated in vacuo, and the residue was filtered through Celite. Bis(di-tert-butylmethylsilyl)dihydrosilane (4) [2] was obtained in nearly quantitative yield; ${ }^{1} \mathrm{H}$ NMR $\left(298 \mathrm{~K}, \mathrm{C}_{6} \mathrm{D}_{6}, \delta\right) 0.12$ (s, 6 $\mathrm{H}), 1.08(\mathrm{~s}, 36 \mathrm{H}), 3.12(\mathrm{~s}, 2 \mathrm{H}) ;{ }^{13} \mathrm{C}$ NMR $\left(298 \mathrm{~K}, \mathrm{C}_{6} \mathrm{D}_{6}, \delta\right)-6.2\left(\mathrm{SiCH}_{3}\right), 20.1\left(\mathrm{CCH}_{3}\right), 29.1\left(\mathrm{CCH}_{3}\right)$; ${ }^{29} \mathrm{Si}$ NMR $\left(298 \mathrm{~K}, \mathrm{C}_{6} \mathrm{D}_{6}, \delta\right)-120.5(\mathrm{SiH}), 10.4\left(\mathrm{SiMe}^{t} \mathrm{Bu}_{2}\right)$. Similarly, the reaction of 2 with $\mathrm{D}_{2} \mathrm{O}$ at $-50{ }^{\circ} \mathrm{C}$ resulted in the formation of bis(di-tert-butylmethylsilyl)dideuteriosilane $(\mathbf{5}) ;{ }^{1} \mathrm{H}$ NMR $(298 \mathrm{~K}$, 
$\left.\mathrm{C}_{6} \mathrm{D}_{6}, \delta\right) 0.18(\mathrm{~s}, 6 \mathrm{H}), 1.07(\mathrm{~s}, 36 \mathrm{H}) ;{ }^{13} \mathrm{C}$ NMR $\left(298 \mathrm{~K}, \mathrm{C}_{6} \mathrm{D}_{6}, \delta\right)-6.2\left(\mathrm{SiCH}_{3}\right), 20.1\left(\mathrm{CCH}_{3}\right), 29.1$ $\left(\mathrm{CCH}_{3}\right) ;{ }^{29} \mathrm{Si}$ NMR $\left(298 \mathrm{~K}, \mathrm{C}_{6} \mathrm{D}_{6}, \delta\right)-120.5$ (quintet, $\left.J_{\mathrm{SiD}}=24.4 \mathrm{~Hz}, \mathrm{SiD}\right), 10.2\left(\mathrm{SiMe}^{\prime} \mathrm{Bu}_{2}\right)$.

18-Electron Hafnium-Silylene Complex, $\left(\eta-\mathrm{C}_{5} \mathrm{H}_{4} \mathrm{Et}\right)_{2}\left(\mathrm{PMe}_{3}\right) \mathrm{Hf}=\mathrm{Si}\left(\mathrm{SiMe}^{t} \mathrm{Bu}_{2}\right)_{2}(6)$. Dilithiosilane $1(0.394 \mathrm{mmol})$ was reacted with $\left(\eta-\mathrm{C}_{5} \mathrm{H}_{4} \mathrm{Et}_{2} \mathrm{HfCl}_{2}(120 \mathrm{mg}, 0.321 \mathrm{mmol})\right.$ in dry toluene $(2.0 \mathrm{~mL})$ for $15 \mathrm{~min}$ at $-50{ }^{\circ} \mathrm{C}$ to form a dark green solution. An excess $\mathrm{PMe}_{3}(1.0 \mathrm{M}$ in toluene, $0.7 \mathrm{~mL}, 0.70$ mmol) was added to this solution at $-50^{\circ} \mathrm{C}$, and the mixture gradually changed to a dark red solution. After removal of inorganic salts, the solvent was evaporated in vacuo. The residue was purified by recrystallization from pentane at $-30{ }^{\circ} \mathrm{C}$ to give bis(di-tert-butylmethylsilyl)silanediylbis(ethylcyclopentadienyl)trimethylphosphine hafnium(IV) (6) (94 mg, 33\%) as red crystals. Mp $121-123^{\circ} \mathrm{C}(\mathrm{dec}) .{ }^{1} \mathrm{H}$ NMR $\left(298 \mathrm{~K}, \mathrm{C}_{6} \mathrm{D}_{6}, \delta\right) 0.80(\mathrm{~s}, 3 \mathrm{H}), 0.88(\mathrm{~s}, 3 \mathrm{H}), 0.94\left(\mathrm{~d}, J_{\mathrm{PH}}=6.3 \mathrm{~Hz}, 9 \mathrm{H}\right)$, $1.06\left(\mathrm{t}, J_{\mathrm{CH}}=7.5 \mathrm{~Hz}, 3 \mathrm{H}\right), 1.07$ (t, $\left.J_{\mathrm{CH}}=7.5 \mathrm{~Hz}, 3 \mathrm{H}\right), 1.30(\mathrm{~s}, 9 \mathrm{H}), 1.44(\mathrm{~s}, 9 \mathrm{H}), 1.47(\mathrm{~s}, 18 \mathrm{H})$, 1.81-2.09 (m, 4 H), $4.60(\mathrm{~m}, 1 \mathrm{H}), 4.67(\mathrm{~m}, 1 \mathrm{H}), 4.94(\mathrm{~m}, 1 \mathrm{H}), 5.54(\mathrm{~m}, 1 \mathrm{H}), 6.10(\mathrm{~m}, 1 \mathrm{H}), 6.54$ $(\mathrm{m}, 1 \mathrm{H}), 7.41(\mathrm{~m}, 1 \mathrm{H}), 7.66(\mathrm{~m}, 1 \mathrm{H}) ;{ }^{13} \mathrm{C} \operatorname{NMR}\left(298 \mathrm{~K}, \mathrm{C}_{6} \mathrm{D}_{6}, \delta\right) 3.0\left(\mathrm{SiCH}_{3}\right), 5.0\left(\mathrm{SiCH}_{3}\right), 16.1$ $\left(\mathrm{CH}_{2} \mathrm{CH}_{3}\right), 17.3\left(\mathrm{CH}_{2} \mathrm{CH}_{3}\right), 22.5\left(\mathrm{~d}, J_{\mathrm{PC}}=22.7 \mathrm{~Hz}, \mathrm{PCH}\right), 23.4\left(\mathrm{CH}_{2} \mathrm{CH}_{3}\right), 23.6\left(\mathrm{CH}_{2} \mathrm{CH}_{3}\right), 24.2$ $\left(\mathrm{CCH}_{3}\right), 24.4\left(\mathrm{CCH}_{3}\right), 24.5\left(\mathrm{CCH}_{3}\right), 24.6\left(\mathrm{CCH}_{3}\right), 32.2\left(\mathrm{CCH}_{3}\right), 32.3\left(\mathrm{CCH}_{3}\right), 32.7\left(\mathrm{CCH}_{3}\right), 33.1$ $\left(\mathrm{CCH}_{3}\right), 90.2(\mathrm{Cp}), 95.9(\mathrm{Cp}), 97.7(\mathrm{Cp}), 100.3(\mathrm{Cp}), 102.1(\mathrm{Cp}), 102.6(\mathrm{Cp}), 104.4(\mathrm{Cp}), 108.2(\mathrm{Cp})$, $120.3\left(\mathrm{Cp}_{\text {ipso }}\right), 120.5\left(\mathrm{Cp}_{\text {ipso }}\right) ;{ }^{29} \mathrm{Si}$ NMR $\left(298 \mathrm{~K}, \mathrm{C}_{6} \mathrm{D}_{6}, \delta\right) 16.8\left(\mathrm{~d},{ }^{3} \mathrm{JPS}_{\mathrm{Pi}}=2.6 \mathrm{~Hz}, S i \mathrm{Me}^{t} \mathrm{Bu}_{2}\right), 24.7(\mathrm{~d}$, $\left.{ }^{3} J_{\mathrm{PSi}}=4.5 \mathrm{~Hz}, S i \mathrm{Me}^{\prime} \mathrm{Bu}_{2}\right), 295.4\left(\mathrm{~d},{ }^{2} J_{\mathrm{PSi}}=15.0 \mathrm{~Hz}, \mathrm{Si}=\mathrm{Hf}\right) ;{ }^{31} \mathrm{P} \mathrm{NMR}\left(298 \mathrm{~K}, \mathrm{C}_{6} \mathrm{D}_{6}, \delta\right)-8.6$; UV/Vis (hexane) $\lambda_{\max } / \mathrm{nm}(\varepsilon) 261$ (13300), 327 (2300), 513 (3800).

The single crystals of $\mathbf{6}$ for X-ray diffraction analysis were grown from a pentane solution at $-30{ }^{\circ} \mathrm{C}$. Diffraction data were collected at $120 \mathrm{~K}$ on a Mac Science DIP2030 Image Plate Diffractometer with a rotating anode $(50 \mathrm{kV}, 90 \mathrm{~mA})$ employing graphite-monochromatized Mo- $K \alpha$ radiation $(\lambda=0.71070 \AA)$. The structures were solved by the direct method, using SIR-92 [2] 
program, and refined by the full-matrix least-squares method by SHELXL-97 program [3]. Crystal data for 6 at $120 \mathrm{~K}: \mathrm{C}_{35} \mathrm{H}_{69} \mathrm{Hf} \mathrm{PSi}_{3}, \mathrm{MW}=783.63$, monoclinic, space group $P 2_{1} / \mathrm{c}, Z=4$, $a=$ $19.1080(13), b=11.7660(9), c=19.6560(14) \AA, \beta=118.138(4)^{\circ}, V=3896.9(5) \AA^{3}, D_{\text {calcd }}=1.336 \mathrm{~g}$ $\mathrm{cm}^{-3}, R_{1}(I>2 \sigma(I))=0.0384, w R_{2}$ (all data) $=0.1017$ for 8638 reflections and 380 parameters, GOF $=1.031$.

\section{References}

[1] Sekiguchi, A.; Izumi, R.; Lee, V. Ya.; Ichinohe, M. J. Am. Chem. Soc. 2002, 124, 14822.

[2] Sekiguchi, A.; Fukawa, T.; Nakamoto, M.; Lee, V. Ya.; Ichinohe, M. J. Am. Chem. Soc. 2002, 124, 9865 .

[3] Altomare, A.; Cascarano, G.; Giacovazzo, C.; Guagliardi, A.; Burla, M. C.; Polidori, G.; Camalli, M. J. Appl. Crystallogr. 1994, 27, 435.

[4] Sheldrick, G. M. SHELXL-97, Program for Crystal Structure Refinement, University of Göttingen, Germany, 1997. 


\section{Atomic Coordinations for $\left(\eta-\mathrm{C}_{5} \mathrm{H}_{4} \mathrm{Et}\right)_{2} \mathrm{Hf}=\mathrm{Si}\left(\mathrm{SiMe}_{3}\right)_{2}(7)$ at B3PW91/6-31G(d) Level, LanL2DZ for $\mathrm{Hf}$}

Energy: -1700.91601601 hartree

\begin{tabular}{|c|c|c|c|c|c|c|c|}
\hline Si 1 & -1.599352 & -0.048647 & -0.030506 & C 31 & 2.836987 & -1.758882 & -0.134165 \\
\hline Hf 2 & 0.944677 & -0.045307 & -0.133157 & C 32 & 2.184311 & -1.762442 & 1.132526 \\
\hline Si 3 & -3.084464 & -1.859746 & 0.431335 & C 33 & 0.847931 & -2.228705 & 0.964452 \\
\hline C 4 & -2.359178 & -3.360584 & 1.361265 & C 34 & 4.309638 & -1.539484 & -0.354792 \\
\hline C 5 & -4.527684 & -1.321451 & 1.555179 & C 35 & 4.730053 & -1.276020 & -1.798386 \\
\hline C 6 & -3.811618 & -2.561071 & -1.185266 & H 36 & -0.241371 & -2.859849 & -0.893156 \\
\hline Si 7 & -3.143710 & 1.735181 & -0.399276 & H 37 & 2.042609 & -2.254471 & -2.161174 \\
\hline C 8 & -2.515763 & 3.210461 & -1.435780 & H 38 & 2.658223 & -1.525276 & 2.079637 \\
\hline $\mathrm{C} 9$ & -4.675129 & 1.163097 & -1.38006 & H 39 & 0.127834 & -2.398409 & 1.750528 \\
\hline C 10 & -3.733600 & 2.472541 & 1.256465 & $\mathrm{H} 40$ & 4.826502 & -2.443127 & 0.001068 \\
\hline H 11 & -1.986804 & -3.083252 & 2.354663 & H 41 & 4.664666 & -0.729821 & 0.295075 \\
\hline H 12 & -3.166707 & -4.089474 & 1.512326 & $\mathrm{H} 42$ & 4.258770 & -0.372630 & -2.199389 \\
\hline H 13 & -1.554729 & -3.874142 & 0.825731 & $\mathrm{H} 43$ & 4.460617 & -2.113241 & -2.451417 \\
\hline H 14 & -4.154422 & -1.006191 & 2.537044 & H 44 & 5.815371 & -1.145880 & -1.863370 \\
\hline H 15 & -5.113053 & -0.493949 & 1.143997 & C 45 & 0.636302 & 2.400935 & 0.085652 \\
\hline H 16 & -5.210905 & -2.166236 & 1.715377 & C 46 & 1.890204 & 2.134526 & 0.709468 \\
\hline H 17 & -3.020390 & -2.965455 & -1.828224 & C 47 & 2.762849 & 1.644611 & -0.295799 \\
\hline H 18 & -4.519671 & -3.373738 & -0.974732 & C 48 & 2.060606 & 1.613824 & -1.535164 \\
\hline H 19 & -4.339723 & -1.794699 & -1.761929 & C 49 & 0.745603 & 2.102206 & -1.306760 \\
\hline H 20 & -2.219397 & 2.896605 & -2.443787 & C 50 & 2.198804 & 2.365046 & 2.168365 \\
\hline H 21 & -3.342865 & 3.923923 & -1.550696 & C 51 & 3.618599 & 1.999523 & 2.592464 \\
\hline Н 22 & -1.678338 & 3.755489 & -0.989134 & H 52 & -0.225567 & 2.825819 & 0.580254 \\
\hline H 23 & -4.382065 & 0.807998 & -2.375360 & H 53 & 3.795309 & 1.354061 & -0.149715 \\
\hline $\mathrm{H} 24$ & -5.233367 & 0.357895 & -0.893150 & H 54 & 2.476078 & 1.321267 & -2.492580 \\
\hline H 25 & -5.362722 & 2.008121 & -1.519136 & H 55 & -0.021316 & 2.255174 & -2.050927 \\
\hline H 26 & -4.186682 & 1.712691 & 1.901938 & H 56 & 1.473562 & 1.820978 & 2.790736 \\
\hline H 27 & -2.895894 & 2.913309 & 1.810521 & H 57 & 2.021659 & 3.427631 & 2.385576 \\
\hline H 28 & -4.477906 & 3.262533 & 1.089596 & H 58 & 4.363131 & 2.580884 & 2.037215 \\
\hline C 29 & 0.650831 & -2.468873 & -0.426987 & H 59 & 3.829740 & 0.936596 & 2.426821 \\
\hline C 30 & 1.870860 & -2.160521 & -1.094815 & H 60 & 3.764837 & 2.203532 & 3.658139 \\
\hline
\end{tabular}


NPA Charge Distributions for $\left(\eta-\mathrm{C}_{5} \mathrm{H}_{4} \mathrm{Et}\right) \mathrm{Hf}=\mathrm{Si}\left(\mathrm{SiMe}_{3}\right)_{2}$ (7) at B3LYP/6-31G(d) Level, LanL2DZ for Hf

$\begin{array}{llllll}\text { Si 1 } & -0.39567 & \text { H 21 } & 0.24443 & \text { H 41 } & 0.23778 \\ \text { Hf 2 } & 1.22543 & \text { H 22 } & 0.23628 & \text { H 42 } & 0.22363 \\ \text { Si 3 } & 1.39622 & \text { H 23 } & 0.24480 & \text { H 43 } & 0.23183 \\ \text { C 4 } & -1.17937 & \text { H 24 } & 0.24204 & \text { H 44 } & 0.24096 \\ \text { C 5 } & -1.17876 & \text { H 25 } & 0.24121 & \text { C 45 } & -0.34959 \\ \text { C 6 } & -1.18224 & \text { H 26 } & 0.24795 & \text { C 46 } & -0.14655 \\ \text { Si 7 } & 1.39640 & \text { H 27 } & 0.23955 & \text { C 47 } & -0.34652 \\ \text { C 8 } & -1.17859 & \text { H 28 } & 0.23980 & \text { C 48 } & -0.39367 \\ \text { C 9 } & -1.17934 & \text { C 29 } & -0.35318 & \text { C 49 } & -0.36211 \\ \text { C 10 } & -1.18239 & \text { C 30 } & -0.36865 & \text { C 50 } & -0.46738 \\ \text { H 11 } & 0.23843 & \text { C 31 } & -0.11717 & \text { C 51 } & -0.67396 \\ \text { H 12 } & 0.24484 & \text { C 32 } & -0.38820 & \text { H 52 } & 0.27779 \\ \text { H 13 } & 0.23711 & \text { C 33 } & -0.37066 & \text { H 53 } & 0.26297 \\ \text { H 14 } & 0.24455 & \text { C 34 } & -0.46399 & \text { H 54 } & 0.26641 \\ \text { H 15 } & 0.24166 & \text { C 35 } & -0.67233 & \text { H 55 } & 0.28286 \\ \text { H 16 } & 0.24112 & \text { H 36 } & 0.28137 & \text { H 56 } & 0.25049 \\ \text { H 17 } & 0.23994 & \text { H 37 } & 0.26791 & \text { H 57 } & 0.25716 \\ \text { H 18 } & 0.23964 & \text { H 38 } & 0.26453 & \text { H 58 } & 0.23206 \\ \text { H 19 } & 0.24789 & \text { H 39 } & 0.28329 & \text { H 59 } & 0.22069 \\ \text { H 20 } & 0.23904 & \text { H 40 } & 0.25789 & \text { H 60 } & 0.24241\end{array}$

Total $\quad 0.00000$ 
Atomic Coordinations for $\left(\eta-\mathrm{C}_{5} \mathrm{H}_{4} \mathrm{Et}\right)_{2}\left(\mathrm{PMe}_{3}\right) \mathrm{Hf}=\mathrm{Si}\left(\mathrm{SiMe}_{3}\right)_{2}(8)$ at $\mathrm{B3PW91/6-31G(d)} \mathrm{Level,}$ LanL2DZ for $\mathbf{H f}$

Energy: -2161.61221485 hartree

\begin{tabular}{|c|c|c|c|c|c|c|c|}
\hline Si 1 & -1.632012 & -0.064284 & -0.244200 & H 32 & -3.825821 & 2.882717 & 2.517567 \\
\hline Hf 2 & 0.907605 & 0.237292 & -0.004300 & H 33 & 0.158282 & -3.366853 & 3.152989 \\
\hline Si 3 & -2.830349 & -1.725448 & -1.479337 & H 34 & 0.584580 & -3.468751 & 1.427371 \\
\hline $\mathrm{C} 4$ & -3.018114 & -3.346245 & -0.486017 & H 35 & -0.968490 & -2.762234 & 1.898433 \\
\hline $\mathrm{C} 5$ & -4.594909 & -1.207265 & -1.985409 & Н 36 & -1.124808 & -0.223429 & 3.356889 \\
\hline $\mathrm{C} 6$ & -2.048807 & -2.204293 & -3.154795 & Н 37 & 0.369126 & 0.479772 & 4.013141 \\
\hline Si 7 & -3.40921 & 1.136923 & 0.795335 & H 38 & -0.133369 & -1.147131 & 4.523290 \\
\hline C 8 & -4.452046 & 2.137674 & -0.447779 & H 39 & 2.261860 & -2.150717 & 4.049228 \\
\hline C 9 & -4.563165 & -0.066339 & 1.726491 & H 40 & 2.943122 & -0.639008 & 3.404287 \\
\hline C 10 & -2.913392 & 2.410578 & 2.129271 & H 41 & 3.091681 & -2.135955 & 2.474549 \\
\hline P 11 & 0.831712 & -1.176439 & 2.266553 & $\mathrm{C} 42$ & 2.990011 & -0.850126 & -0.989838 \\
\hline C 12 & 0.082054 & -2.859349 & 2.184025 & C 43 & 2.265657 & -0.211981 & -2.037250 \\
\hline C 13 & -0.105239 & -0.448036 & 3.679683 & C 44 & 1.021755 & -0.873740 & -2.216067 \\
\hline C 14 & 2.430133 & -1.567976 & 3.135831 & C 45 & 0.959576 & -1.923902 & -1.256760 \\
\hline H 15 & -2.047361 & -3.810096 & -0.273983 & C 46 & 2.170234 & -1.905558 & -0.513199 \\
\hline H 16 & -3.619447 & -4.076265 & -1.044284 & C 47 & 4.420099 & -0.596762 & -0.598611 \\
\hline H 17 & -3.515582 & -3.168992 & 0.474907 & C 48 & 5.379859 & -1.615031 & -1.229120 \\
\hline H 18 & -5.084642 & -2.047381 & -2.49609 & H 49 & 2.631550 & 0.620020 & -2.626920 \\
\hline H 19 & -4.569144 & -0.365472 & -2.686872 & H 50 & 0.276486 & -0.64488 & -2.963073 \\
\hline H 20 & -5.228027 & -0.918688 & -1.141206 & H 51 & 0.154303 & -2.635475 & -1.144629 \\
\hline H 21 & -1.950148 & -1.327626 & -3.806342 & H 52 & 2.455444 & -2.622499 & 0.247402 \\
\hline H 22 & -1.066247 & -2.679236 & -3.077872 & H 53 & 4.529036 & -0.624041 & 0.493466 \\
\hline H 23 & -2.716147 & -2.913432 & -3.662765 & H 54 & 4.714530 & 0.411119 & -0.914721 \\
\hline H 24 & -3.834759 & 2.885826 & -0.959694 & H 55 & 5.143662 & -2.634139 & -0.903577 \\
\hline H 25 & -5.261271 & 2.670423 & 0.069495 & H 56 & 6.417480 & -1.401084 & -0.947453 \\
\hline H 26 & -4.903883 & 1.504203 & -1.216288 & H 57 & 5.310957 & -1.591958 & -2.322130 \\
\hline H 27 & -5.429304 & 0.467568 & 2.139765 & C 58 & 1.404497 & 2.684742 & -0.544836 \\
\hline H 28 & -4.031046 & -0.534131 & 2.564111 & C 59 & 2.44831 & 2.199587 & 0.294418 \\
\hline Н 29 & -4.939660 & -0.873139 & 1.089606 & C 60 & 1.911813 & 1.885307 & 1.566795 \\
\hline H 30 & -2.405316 & 1.946190 & 2.982378 & C 61 & 0.515258 & 2.168777 & 1.521232 \\
\hline 31 & -2.271376 & 3.212126 & 1.748258 & $\mathrm{C} 62$ & 0.210072 & 2.661848 & 0.225252 \\
\hline
\end{tabular}




$\begin{array}{llllllll}\text { C 63 } & 1.586050 & 3.288910 & -1.913207 & \text { H 69 } & 2.445958 & 2.818856 & -2.407061 \\ \text { C 64 } & 0.363752 & 3.218631 & -2.827142 & \text { H 70 } & 1.870674 & 4.343331 & -1.777916 \\ \text { H 65 } & 3.489099 & 2.111902 & 0.006262 & \text { H 71 } & 0.033672 & 2.184615 & -2.971654 \\ \text { H 66 } & 2.478207 & 1.549832 & 2.426868 & \text { H 72 } & 0.592128 & 3.653139 & -3.806699 \\ \text { H 67 } & -0.184813 & 2.076578 & 2.337924 & \text { H 73 } & -0.481326 & 3.775550 & -2.408294 \\ \text { H 68 } & -0.761805 & 2.993029 & -0.110355 & & & & \end{array}$


NPA Charge Distributions for $\left(\eta-\mathrm{C}_{5} \mathrm{H}_{4} \mathrm{Et}\right)_{2}\left(\mathrm{PMe}_{3}\right) \mathrm{Hf}=\mathrm{Si}\left(\mathrm{SiMe}_{3}\right)_{2}(8)$ at $\mathrm{B3LYP} / 6-31 \mathrm{G}(\mathrm{d})$ Level, LanL2DZ for Hf

\begin{tabular}{|c|c|c|c|c|c|c|c|c|}
\hline $\mathrm{Si}$ & 1 & 0.34035 & $\mathrm{H}$ & 26 & 0.24607 & $\mathrm{H}$ & 51 & 0.27125 \\
\hline $\mathrm{Hf}$ & 2 & 0.78398 & $\mathrm{H}$ & 27 & 0.24063 & $\mathrm{H}$ & 52 & 0.25302 \\
\hline $\mathrm{Si}$ & 3 & 1.38145 & $\mathrm{H}$ & 28 & 0.23918 & $\mathrm{H}$ & 53 & 0.23674 \\
\hline $\mathrm{C}$ & 4 & -1.18162 & $\mathrm{H}$ & 29 & 0.24109 & $\mathrm{H}$ & 54 & 0.23600 \\
\hline $\mathrm{C}$ & 5 & -1.18338 & $\mathrm{H}$ & 30 & 0.23287 & $\mathrm{H}$ & 55 & 0.22819 \\
\hline $\mathrm{C}$ & 6 & -1.17980 & $\mathrm{H}$ & 31 & 0.24051 & $\mathrm{H}$ & 56 & 0.23154 \\
\hline $\mathrm{Si}$ & 7 & 1.38121 & $\mathrm{H}$ & 32 & 0.24364 & $\mathrm{H}$ & 57 & 0.23302 \\
\hline $\mathrm{C}$ & 8 & -1.18081 & $\mathrm{H}$ & 33 & 0.24117 & $\mathrm{C}$ & 58 & -0.13107 \\
\hline $\mathrm{C}$ & 9 & -1.18234 & $\mathrm{H}$ & 34 & 0.25133 & $\mathrm{C}$ & 59 & -0.32523 \\
\hline $\mathrm{C}$ & 10 & -1.17765 & $\mathrm{H}$ & 35 & 0.27420 & $\mathrm{C}$ & 60 & -0.36392 \\
\hline$P$ & 11 & 1.03478 & $\mathrm{H}$ & 36 & 0.26776 & $\mathrm{C}$ & 61 & -0.36142 \\
\hline $\mathrm{C}$ & 12 & -1.00102 & $\mathrm{H}$ & 37 & 0.25249 & $\mathrm{C}$ & 62 & -0.30032 \\
\hline C & 13 & -1.00174 & $\mathrm{H}$ & 38 & 0.24451 & $\mathrm{C}$ & 63 & -0.45667 \\
\hline $\mathrm{C}$ & 14 & -1.01094 & $\mathrm{H}$ & 39 & 0.24350 & $\mathrm{C}$ & 64 & -0.67111 \\
\hline $\mathrm{H}$ & 15 & 0.23505 & $\mathrm{H}$ & 40 & 0.24887 & $\mathrm{H}$ & 65 & 0.25783 \\
\hline $\mathrm{H}$ & 16 & 0.24058 & $\mathrm{H}$ & 41 & 0.24818 & $\mathrm{H}$ & 66 & 0.25868 \\
\hline $\mathrm{H}$ & 17 & 0.24320 & $\mathrm{C}$ & 42 & -0.13949 & $\mathrm{H}$ & 67 & 0.26954 \\
\hline $\mathrm{H}$ & 18 & 0.24046 & $\mathrm{C}$ & 43 & -0.34277 & $\mathrm{H}$ & 68 & 0.27331 \\
\hline $\mathrm{H}$ & 19 & 0.24444 & $\mathrm{C}$ & 44 & -0.33521 & $\mathrm{H}$ & 69 & 0.23133 \\
\hline $\mathrm{H}$ & 20 & 0.24101 & $\mathrm{C}$ & 45 & -0.34370 & $\mathrm{H}$ & 70 & 0.25103 \\
\hline $\mathrm{H}$ & 21 & 0.24229 & $\mathrm{C}$ & 46 & -0.32838 & $\mathrm{H}$ & 71 & 0.23767 \\
\hline $\mathrm{H}$ & 22 & 0.23909 & $\mathrm{C}$ & 47 & -0.45693 & $\mathrm{H}$ & 72 & 0.23366 \\
\hline $\mathrm{H}$ & 23 & 0.24226 & $\mathrm{C}$ & 48 & -0.66222 & $\mathrm{H}$ & 73 & 0.22971 \\
\hline $\mathrm{H}$ & 24 & 0.24179 & $\mathrm{H}$ & 49 & 0.26060 & & & \\
\hline $\mathrm{H}$ & 25 & 0.23785 & $\mathrm{H}$ & 50 & 0.27951 & & & \\
\hline
\end{tabular}

Total $\quad 0.00000$ 
Table 1. Crystal data and structure refinement for $\left(\eta-\mathrm{C}_{5} \mathrm{H}_{4} \mathrm{Et}\right)_{2}\left(\mathrm{PMe}_{3}\right) \mathrm{Hf}=\mathrm{Si}\left(\mathrm{SiMe}^{\prime} \mathrm{Bu}_{2}\right)_{2}(6)$.

\begin{tabular}{|c|c|c|}
\hline Identification code & \multicolumn{2}{|l|}{$\mathrm{Me}_{3} \mathrm{P}-\mathrm{Hf}=\mathrm{Si}$} \\
\hline Empirical formula & \multicolumn{2}{|l|}{ C35 H69 Hf P Si3 } \\
\hline Formula weight & \multicolumn{2}{|l|}{783.63} \\
\hline Temperature & \multicolumn{2}{|l|}{$120(2) \mathrm{K}$} \\
\hline Wavelength & \multicolumn{2}{|l|}{$0.71070 \AA$} \\
\hline Crystal system & \multicolumn{2}{|l|}{ monoclinic } \\
\hline Space group & \multicolumn{2}{|l|}{$P 2_{1} / \mathrm{c}$} \\
\hline \multirow[t]{3}{*}{ Unit cell dimensions } & $a=19.1080(13) \AA$ & $\alpha=90^{\circ}$ \\
\hline & $b=11.7660(9) \AA$ & $\beta=118.138(4)^{\circ}$. \\
\hline & $c=19.6560(14) \AA$ & $\gamma=90^{\circ}$ \\
\hline Volume & \multicolumn{2}{|l|}{$3896.9(5) \AA^{3}$} \\
\hline $\mathrm{Z}$ & \multicolumn{2}{|l|}{4} \\
\hline Density (calculated) & \multicolumn{2}{|l|}{$1.336 \mathrm{Mg} / \mathrm{m}^{3}$} \\
\hline Absorption coefficient & \multicolumn{2}{|l|}{$2.832 \mathrm{~mm}^{-1}$} \\
\hline $\mathrm{F}(000)$ & \multicolumn{2}{|l|}{1632} \\
\hline Crystal size & \multicolumn{2}{|c|}{$0.35 \times 0.25 \times 0.25 \mathrm{~mm}^{3}$} \\
\hline Theta range for data collection & \multicolumn{2}{|l|}{2.09 to $28.02^{\circ}$. } \\
\hline Index ranges & \multicolumn{2}{|c|}{$0<=\mathrm{h}<=25,0<=\mathrm{k}<=15,-25<=\mathrm{l}<=22$} \\
\hline Reflections collected & \multicolumn{2}{|l|}{8638} \\
\hline Independent reflections & \multicolumn{2}{|c|}{$8638[R($ int $)=0.042]$} \\
\hline Completeness to theta $=28.02^{\circ}$ & \multicolumn{2}{|l|}{$91.5 \%$} \\
\hline Max. and min. transmission & \multicolumn{2}{|l|}{0.5379 and 0.4373} \\
\hline Refinement method & \multicolumn{2}{|c|}{ Full-matrix least-squares on $\mathrm{F}^{2}$} \\
\hline Data / restraints / parameters & \multicolumn{2}{|l|}{$8638 / 0 / 380$} \\
\hline Goodness-of-fit on $\mathrm{F}^{2}$ & \multicolumn{2}{|l|}{1.031} \\
\hline Final $R$ indices $[\mathrm{I}>2 \operatorname{sigma}(\mathrm{I})]$ & \multicolumn{2}{|c|}{$R_{1}=0.0384, \mathrm{w} R_{2}=0.0984$} \\
\hline $\mathrm{R}$ indices (all data) & \multicolumn{2}{|c|}{$R_{1}=0.0438, \mathrm{w} R_{2}=0.1017$} \\
\hline
\end{tabular}




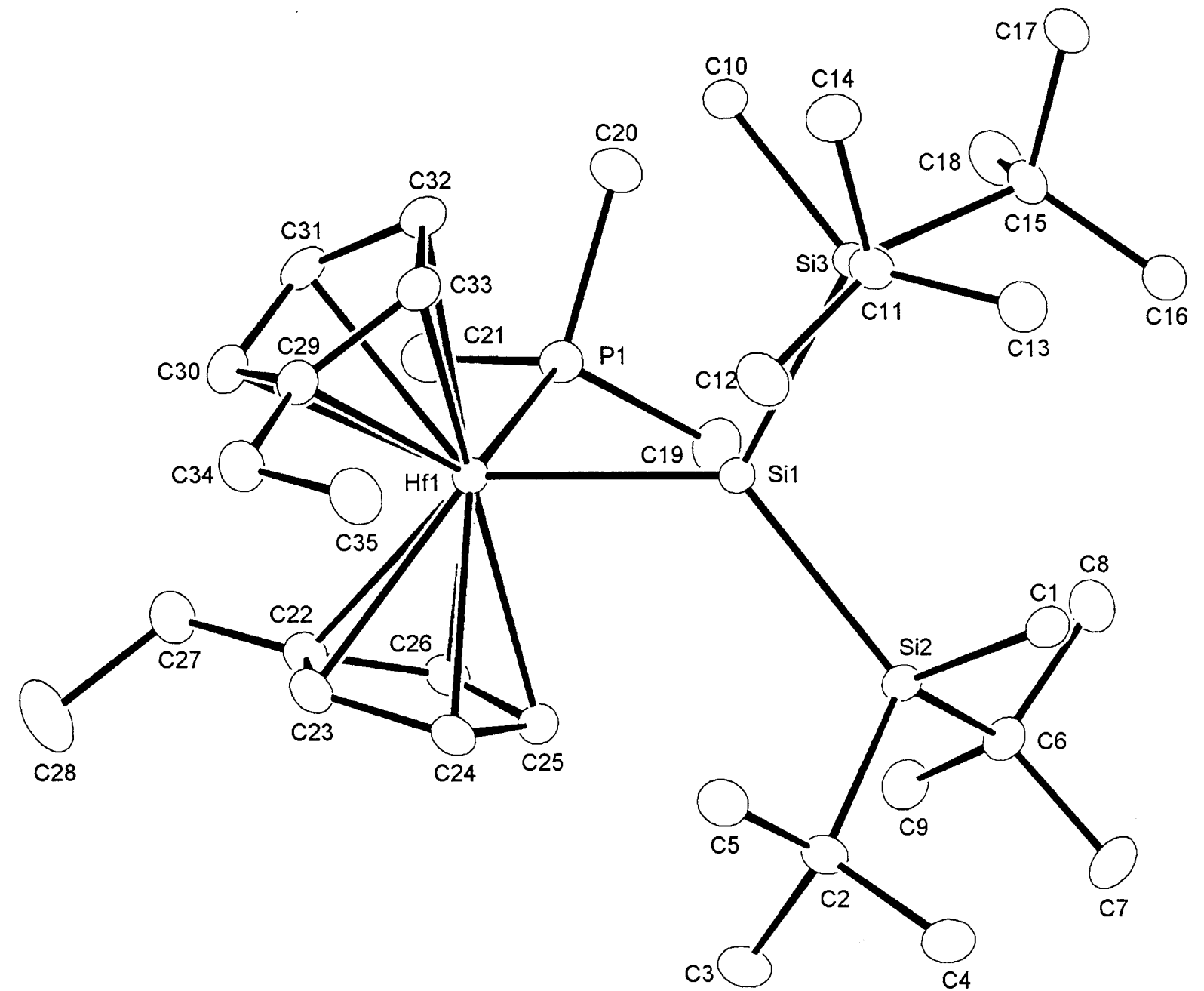

Figure 2. ORTEP drawing of bis[di-tert-butyl(methyl)silyl]silanediylbis(ethylcyclopentadienyl)trimethylphosphine hafnium (IV) (6) (30\% thermal ellipsoids). Hydrogen atoms are omitted for clarity. 
Table 2. Atomic coordinates $\left(\times 10^{4}\right)$ and equivalent isotropic displacement parameters $\left(\AA^{2} \times 10^{3}\right)$ for $\left(\eta-\mathrm{C}_{5} \mathrm{H}_{4} \mathrm{Et}\right)_{2^{-}}$ $\left(\mathrm{PMe}_{3}\right) \mathrm{Hf}=\mathrm{Si}\left(\mathrm{SiMe}^{\prime} \mathrm{Bu}_{2}\right)_{2}(6)$. U(eq) is defined as one third of the trace of the orthogonalized $\mathrm{U}^{\mathrm{ij}}$ tensor.

\begin{tabular}{|c|c|c|c|c|}
\hline & $\mathrm{x}$ & $\mathrm{y}$ & $z$ & $\mathrm{U}(\mathrm{eq})$ \\
\hline $\operatorname{Si}(1)$ & $7444(1)$ & $1033(1)$ & $7085(1)$ & $21(1)$ \\
\hline $\mathrm{Hf}(1)$ & $8512(1)$ & $631(1)$ & $6613(1)$ & $21(1)$ \\
\hline $\mathrm{Si}(2)$ & $6730(1)$ & $2810(1)$ & $6980(1)$ & $25(1)$ \\
\hline$C(1)$ & $6311(2)$ & $2712(3)$ & $7674(2)$ & $31(1)$ \\
\hline$C(2)$ & $7374(2)$ & $4192(3)$ & $7363(2)$ & $32(1)$ \\
\hline$C(3)$ & $7558(3)$ & $4830(3)$ & $6772(3)$ & $40(1)$ \\
\hline $\mathrm{C}(4)$ & $6958(3)$ & $5074(3)$ & $7635(3)$ & $43(1)$ \\
\hline$C(5)$ & $8161(2)$ & $3878(3)$ & $8080(2)$ & $37(1)$ \\
\hline$C(6)$ & $5811(2)$ & $3074(3)$ & $5972(2)$ & $31(1)$ \\
\hline$C(7)$ & $5358(3)$ & $4159(4)$ & $5958(3)$ & $42(1)$ \\
\hline$C(8)$ & $5234(2)$ & 2071(4) & $5805(2)$ & $38(1)$ \\
\hline$C(9)$ & $6009(2)$ & $3149(4)$ & $5301(2)$ & $35(1)$ \\
\hline $\mathrm{Si}(3)$ & $7098(1)$ & $-336(1)$ & $7828(1)$ & $23(1)$ \\
\hline$C(10)$ & $7630(2)$ & $-1722(3)$ & $7864(2)$ & $31(1)$ \\
\hline$C(11)$ & $7510(2)$ & $50(3)$ & $8926(2)$ & $29(1)$ \\
\hline$C(12)$ & $8211(3)$ & $879(4)$ & $9166(2)$ & $38(1)$ \\
\hline$C(13)$ & $6912(3)$ & $566(3)$ & $9148(2)$ & $35(1)$ \\
\hline$C(14)$ & $7835(3)$ & $-1027(4)$ & $9431(2)$ & $37(1)$ \\
\hline$C(15)$ & $6003(2)$ & $-898(3)$ & $7370(2)$ & $28(1)$ \\
\hline$C(16)$ & $5369(2)$ & $-31(3)$ & $7293(2)$ & $34(1)$ \\
\hline$C(17)$ & $5937(2)$ & $-1913(3)$ & $7841(2)$ & $36(1)$ \\
\hline$C(18)$ & $5770(2)$ & $-1345(4)$ & $6556(2)$ & $40(1)$ \\
\hline $\mathrm{P}(1)$ & $7451(1)$ & $-512(1)$ & $5393(1)$ & $28(1)$ \\
\hline$C(19)$ & $6478(2)$ & $139(4)$ & $4787(2)$ & $38(1)$ \\
\hline$C(20)$ & $7151(2)$ & $-1911(3)$ & $5565(2)$ & $35(1)$ \\
\hline$C(21)$ & $7755(3)$ & $-885(4)$ & $4661(2)$ & $37(1)$ \\
\hline$C(22)$ & $9047(2)$ & $1648(3)$ & $5813(2)$ & $29(1)$ \\
\hline $\mathrm{C}(23)$ & $9377(2)$ & 2151(3) & $6552(2)$ & $30(1)$ \\
\hline
\end{tabular}




\begin{tabular}{lrrrr}
$\mathrm{C}(24)$ & $8768(2)$ & $2673(3)$ & $6654(2)$ & $31(1)$ \\
$\mathrm{C}(25)$ & $8044(2)$ & $2483(3)$ & $5977(2)$ & $30(1)$ \\
$\mathrm{C}(26)$ & $8215(2)$ & $1864(3)$ & $5460(2)$ & $29(1)$ \\
$\mathrm{C}(27)$ & $9489(2)$ & $1117(4)$ & $5432(2)$ & $38(1)$ \\
$\mathrm{C}(28)$ & $10207(4)$ & $1799(6)$ & $5558(5)$ & $95(3)$ \\
$\mathrm{C}(29)$ & $9816(2)$ & $40(3)$ & $7771(2)$ & $31(1)$ \\
$\mathrm{C}(30)$ & $9835(2)$ & $-326(3)$ & $7093(2)$ & $34(1)$ \\
$\mathrm{C}(31)$ & $9238(2)$ & $-1146(3)$ & $6713(2)$ & $32(1)$ \\
$\mathrm{C}(32)$ & $8833(2)$ & $-1303(3)$ & $7153(2)$ & $32(1)$ \\
$\mathrm{C}(33)$ & $9194(2)$ & $-564(3)$ & $7807(2)$ & $29(1)$ \\
$\mathrm{C}(34)$ & $10412(2)$ & $813(4)$ & $8382(2)$ & $36(1)$ \\
$\mathrm{C}(35)$ & $10127(2)$ & $1316(4)$ & $8919(2)$ & $41(1)$ \\
& & & & \\
\hline
\end{tabular}


Table 3. Bond lengths $[\AA]$ and angles $\left[^{\circ}\right]$ for $\left(\eta-\mathrm{C}_{5} \mathrm{H}_{4} \mathrm{Et}\right)_{2}\left(\mathrm{PMe}_{3}\right) \mathrm{Hf}=\mathrm{Si}\left(\mathrm{SiMe}^{\prime} \mathrm{Bu}_{2}\right)_{2}(6)$.

\begin{tabular}{|c|c|c|c|}
\hline $\operatorname{Si}(1)-\operatorname{Si}(2)$ & $2.4512(13)$ & $C(15)-C(17)$ & $1.552(5)$ \\
\hline $\operatorname{Si}(1)-\operatorname{Si}(3)$ & $2.4643(13)$ & $P(1)-C(20)$ & $1.827(4)$ \\
\hline $\operatorname{Si}(1)-H f(1)$ & $2.6515(9)$ & $P(1)-C(19)$ & $1.836(4)$ \\
\hline $\mathrm{Hf}(1)-\mathrm{C}(24)$ & $2.446(4)$ & $P(1)-C(21)$ & $1.841(4)$ \\
\hline $\mathrm{Hf}(1)-\mathrm{C}(25)$ & $2.461(3)$ & $\mathrm{C}(22)-\mathrm{C}(23)$ & $1.412(5)$ \\
\hline $\mathrm{Hf}(1)-\mathrm{C}(32)$ & $2.463(4)$ & $C(22)-C(26)$ & $1.425(5)$ \\
\hline $\mathrm{Hf}(1)-\mathrm{C}(31)$ & $2.465(3)$ & $\mathrm{C}(22)-\mathrm{C}(27)$ & $1.503(5)$ \\
\hline $\mathrm{Hf}(1)-\mathrm{C}(23)$ & $2.477(4)$ & $\mathrm{C}(23)-\mathrm{C}(24)$ & $1.411(5)$ \\
\hline $\mathrm{Hf}(1)-\mathrm{C}(33)$ & $2.509(3)$ & $\mathrm{C}(24)-\mathrm{C}(25)$ & $1.414(5)$ \\
\hline $\mathrm{Hf}(1)-\mathrm{C}(30)$ & $2.510(4)$ & $C(25)-C(26)$ & $1.409(5)$ \\
\hline $\mathrm{Hf}(1)-\mathrm{C}(26)$ & $2.521(4)$ & $\mathrm{C}(27)-\mathrm{C}(28)$ & $1.506(7)$ \\
\hline $\mathrm{Hf}(1)-\mathrm{C}(22)$ & $2.544(4)$ & $\mathrm{C}(29)-\mathrm{C}(33)$ & $1.414(5)$ \\
\hline $\mathrm{Hf}(1)-\mathrm{C}(29)$ & $2.554(3)$ & $C(29)-C(30)$ & $1.417(5)$ \\
\hline$H f(1)-P(1)$ & $2.6619(9)$ & $\mathrm{C}(29)-\mathrm{C}(34)$ & $1.508(5)$ \\
\hline $\operatorname{Si}(2)-C(1)$ & $1.883(4)$ & $C(30)-C(31)$ & $1.410(6)$ \\
\hline $\operatorname{Si}(2)-C(6)$ & $1.955(4)$ & $C(31)-C(32)$ & $1.419(5)$ \\
\hline $\operatorname{Si}(2)-C(2)$ & $1.963(4)$ & $C(32)-C(33)$ & $1.430(5)$ \\
\hline$C(2)-C(5)$ & $1.544(6)$ & $C(34)-C(35)$ & $1.519(6)$ \\
\hline$C(2)-C(4)$ & $1.548(5)$ & & \\
\hline$C(2)-C(3)$ & $1.556(5)$ & $\operatorname{Si}(2)-\operatorname{Si}(1)-\operatorname{Si}(3)$ & $108.27(5)$ \\
\hline$C(6)-C(9)$ & $1.536(5)$ & $\mathrm{Si}(2)-\mathrm{Si}(1)-\mathrm{Hf}(1)$ & $127.74(4)$ \\
\hline$C(6)-C(7)$ & $1.535(5)$ & $\mathrm{Si}(3)-\mathrm{Si}(1)-\mathrm{Hf}(1)$ & $123.77(4)$ \\
\hline$C(6)-C(8)$ & $1.540(5)$ & $\mathrm{C}(24)-\mathrm{Hf}(1)-\mathrm{C}(25)$ & $33.50(12)$ \\
\hline $\mathrm{Si}(3)-\mathrm{C}(10)$ & $1.905(4)$ & $\mathrm{C}(24)-\mathrm{Hf}(1)-\mathrm{C}(32)$ & $152.65(13)$ \\
\hline $\operatorname{Si}(3)-C(15)$ & $1.963(4)$ & $\mathrm{C}(25)-\mathrm{Hf}(1)-\mathrm{C}(32)$ & $173.67(12)$ \\
\hline $\operatorname{Si}(3)-C(11)$ & $1.971(4)$ & $C(24)-H f(1)-C(31)$ & $137.20(13)$ \\
\hline$C(11)-C(13)$ & $1.530(6)$ & $\mathrm{C}(25)-\mathrm{Hf}(1)-\mathrm{C}(31)$ & $147.87(13)$ \\
\hline $\mathrm{C}(11)-\mathrm{C}(12)$ & $1.539(5)$ & $C(32)-H f(1)-C(31)$ & $33.47(13)$ \\
\hline$C(11)-C(14)$ & $1.547(5)$ & $\mathrm{C}(24)-\mathrm{Hf}(1)-\mathrm{C}(23)$ & $33.32(12)$ \\
\hline$C(15)-C(16)$ & $1.535(5)$ & $C(25)-H f(1)-C(23)$ & $55.05(12)$ \\
\hline$C(15)-C(18)$ & $1.538(5)$ & $\mathrm{C}(32)-\mathrm{Hf}(1)-\mathrm{C}(23)$ & $130.64(13)$ \\
\hline
\end{tabular}




\begin{tabular}{|c|c|c|c|}
\hline$C(31)-H f(1)-C(23)$ & $104.60(13)$ & $\mathrm{C}(30)-\mathrm{Hf}(1)-\mathrm{C}(29)$ & $32.49(12)$ \\
\hline $\mathrm{C}(24)-\mathrm{Hf}(1)-\mathrm{C}(33)$ & $120.83(12)$ & $C(26)-H f(1)-C(29)$ & $131.98(12)$ \\
\hline $\mathrm{C}(25)-\mathrm{Hf}(1)-\mathrm{C}(33)$ & $151.12(12)$ & $C(22)-H f(1)-C(29)$ & $99.84(12)$ \\
\hline $\mathrm{C}(32)-\mathrm{Hf}(1)-\mathrm{C}(33)$ & $33.43(11)$ & $\mathrm{C}(24)-\mathrm{Hf}(1)-\mathrm{Si}(1)$ & $89.36(9)$ \\
\hline $\mathrm{C}(31)-\mathrm{Hf}(1)-\mathrm{C}(33)$ & $54.94(12)$ & $\mathrm{C}(25)-\mathrm{Hf}(1)-\mathrm{Si}(1)$ & $82.24(9)$ \\
\hline $\mathrm{C}(23)-\mathrm{Hf}(1)-\mathrm{C}(33)$ & $112.66(12)$ & $\mathrm{C}(32)-\mathrm{Hf}(1)-\mathrm{Si}(1)$ & $95.21(9)$ \\
\hline $\mathrm{C}(24)-\mathrm{Hf}(1)-\mathrm{C}(30)$ & $106.11(13)$ & $\mathrm{C}(31)-\mathrm{Hf}(1)-\mathrm{Si}(1)$ & $128.37(10)$ \\
\hline $\mathrm{C}(25)-\mathrm{Hf}(1)-\mathrm{C}(30)$ & $130.40(13)$ & $\mathrm{C}(23)-\mathrm{Hf}(1)-\mathrm{Si}(1)$ & $122.00(9)$ \\
\hline $\mathrm{C}(32)-\mathrm{Hf}(1)-\mathrm{C}(30)$ & $54.66(13)$ & $\mathrm{C}(33)-\mathrm{Hf}(1)-\mathrm{Si}(1)$ & $85.40(10)$ \\
\hline $\mathrm{C}(31)-\mathrm{Hf}(1)-\mathrm{C}(30)$ & $32.90(13)$ & $\mathrm{C}(30)-\mathrm{Hf}(1)-\mathrm{Si}(1)$ & $138.90(9)$ \\
\hline$C(23)-H f(1)-C(30)$ & $76.38(13)$ & $\mathrm{C}(26)-\mathrm{Hf}(1)-\mathrm{Si}(1)$ & $109.17(9)$ \\
\hline$C(33)-H f(1)-C(30)$ & $53.83(13)$ & $\mathrm{C}(22)-\mathrm{Hf}(1)-\mathrm{Si}(1)$ & $137.10(9)$ \\
\hline$C(24)-H f(1)-C(26)$ & $54.59(12)$ & $\mathrm{C}(29)-\mathrm{Hf}(1)-\mathrm{Si}(1)$ & $109.00(9)$ \\
\hline$C(25)-\mathrm{Hf}(1)-\mathrm{C}(26)$ & $32.82(12)$ & $C(24)-H f(1)-P(1)$ & $125.53(9)$ \\
\hline $\mathrm{C}(32)-\mathrm{Hf}(1)-\mathrm{C}(26)$ & $145.45(12)$ & $C(25)-H f(1)-P(1)$ & $92.82(9)$ \\
\hline $\mathrm{C}(31)-\mathrm{Hf}(1)-\mathrm{C}(26)$ & $116.00(13)$ & $C(32)-H f(1)-P(1)$ & $81.41(9)$ \\
\hline $\mathrm{C}(23)-\mathrm{Hf}(1)-\mathrm{C}(26)$ & $54.05(11)$ & $\mathrm{C}(31)-\mathrm{Hf}(1)-\mathrm{P}(1)$ & $78.69(9)$ \\
\hline $\mathrm{C}(33)-\mathrm{Hf}(1)-\mathrm{C}(26)$ & $163.86(13)$ & $C(23)-H f(1)-P(1)$ & $124.27(9)$ \\
\hline $\mathrm{C}(30)-\mathrm{Hf}(1)-\mathrm{C}(26)$ & $110.80(13)$ & $C(33)-H f(1)-P(1)$ & $113.49(8)$ \\
\hline $\mathrm{C}(24)-\mathrm{Hf}(1)-\mathrm{C}(22)$ & $54.91(12)$ & $C(30)-H f(1)-P(1)$ & $108.42(10)$ \\
\hline $\mathrm{C}(25)-\mathrm{Hf}(1)-\mathrm{C}(22)$ & $54.89(12)$ & $C(26)-H f(1)-P(1)$ & $74.18(8)$ \\
\hline $\mathrm{C}(32)-\mathrm{Hf}(1)-\mathrm{C}(22)$ & $127.56(12)$ & $C(22)-H f(1)-P(1)$ & $92.15(8)$ \\
\hline $\mathrm{C}(31)-\mathrm{Hf}(1)-\mathrm{C}(22)$ & $94.14(13)$ & $C(29)-H f(1)-P(1)$ & $132.37(9)$ \\
\hline$C(23)-H f(1)-C(22)$ & $32.64(11)$ & $\mathrm{Si}(1)-\mathrm{Hf}(1)-\mathrm{P}(1)$ & $91.19(3)$ \\
\hline $\mathrm{C}(33)-\mathrm{Hf}(1)-\mathrm{C}(22)$ & $131.17(13)$ & $C(1)-S i(2)-C(6)$ & $104.96(17)$ \\
\hline$C(30)-H f(1)-C(22)$ & $79.37(13)$ & $\mathrm{C}(1)-\mathrm{Si}(2)-\mathrm{C}(2)$ & $100.21(17)$ \\
\hline$C(26)-H f(1)-C(22)$ & $32.69(11)$ & $C(6)-S i(2)-C(2)$ & $110.98(17)$ \\
\hline $\mathrm{C}(24)-\mathrm{Hf}(1)-\mathrm{C}(29)$ & $98.17(13)$ & $C(1)-\operatorname{Si}(2)-\operatorname{Si}(1)$ & $107.25(12)$ \\
\hline$C(25)-H f(1)-C(29)$ & $131.44(12)$ & $C(6)-\operatorname{Si}(2)-\operatorname{Si}(1)$ & $114.76(12)$ \\
\hline $\mathrm{C}(32)-\mathrm{Hf}(1)-\mathrm{C}(29)$ & $54.87(12)$ & $C(2)-\operatorname{Si}(2)-\operatorname{Si}(1)$ & $116.80(13)$ \\
\hline $\mathrm{C}(31)-\mathrm{Hf}(1)-\mathrm{C}(29)$ & $54.73(12)$ & $C(5)-C(2)-C(4)$ & $106.4(3)$ \\
\hline $\mathrm{C}(23)-\mathrm{Hf}(1)-\mathrm{C}(29)$ & $81.16(12)$ & $C(5)-C(2)-C(3)$ & $109.4(3)$ \\
\hline $\mathrm{C}(33)-\mathrm{Hf}(1)-\mathrm{C}(29)$ & $32.42(12)$ & $\mathrm{C}(4)-\mathrm{C}(2)-\mathrm{C}(3)$ & $105.6(3)$ \\
\hline
\end{tabular}




\begin{tabular}{|c|c|c|c|}
\hline$C(5)-C(2)-S i(2)$ & $108.4(2)$ & $C(23)-C(22)-C(26)$ & $106.3(3)$ \\
\hline$C(4)-C(2)-\operatorname{Si}(2)$ & $110.9(3)$ & $C(23)-C(22)-C(27)$ & $127.1(3)$ \\
\hline$C(3)-C(2)-S i(2)$ & $115.8(3)$ & $\mathrm{C}(26)-\mathrm{C}(22)-\mathrm{C}(27)$ & $126.2(3)$ \\
\hline$C(9)-C(6)-C(7)$ & $107.5(3)$ & $\mathrm{C}(23)-\mathrm{C}(22)-\mathrm{Hf}(1)$ & $71.1(2)$ \\
\hline$C(9)-C(6)-C(8)$ & $107.6(3)$ & $\mathrm{C}(26)-\mathrm{C}(22)-\mathrm{Hf}(1)$ & $72.8(2)$ \\
\hline$C(7)-C(6)-C(8)$ & $107.0(3)$ & $\mathrm{C}(27)-\mathrm{C}(22)-\mathrm{Hf}(1)$ & $126.6(3)$ \\
\hline$C(9)-C(6)-\operatorname{Si}(2)$ & $114.3(3)$ & $C(24)-C(23)-C(22)$ & $109.2(3)$ \\
\hline$C(7)-C(6)-S i(2)$ & $112.3(3)$ & $C(24)-C(23)-H f(1)$ & $72.1(2)$ \\
\hline$C(8)-C(6)-S i(2)$ & $107.7(2)$ & $C(22)-C(23)-H f(1)$ & $76.3(2)$ \\
\hline$C(10)-\operatorname{Si}(3)-C(15)$ & $99.45(16)$ & $C(23)-C(24)-C(25)$ & $107.7(3)$ \\
\hline$C(10)-\operatorname{Si}(3)-C(11)$ & $103.07(16)$ & $C(23)-C(24)-H f(1)$ & $74.6(2)$ \\
\hline$C(15)-\operatorname{Si}(3)-C(11)$ & $111.63(16)$ & $C(25)-C(24)-H f(1)$ & $73.9(2)$ \\
\hline$C(10)-\operatorname{Si}(3)-\operatorname{Si}(1)$ & $107.08(13)$ & $C(26)-C(25)-C(24)$ & $107.6(3)$ \\
\hline$C(15)-\operatorname{Si}(3)-\operatorname{Si}(1)$ & $118.33(11)$ & $C(26)-C(25)-H f(1)$ & $75.9(2)$ \\
\hline$C(11)-\operatorname{Si}(3)-\operatorname{Si}(1)$ & $114.72(11)$ & $C(24)-C(25)-H f(1)$ & $72.7(2)$ \\
\hline$C(13)-C(11)-C(12)$ & $108.7(3)$ & $C(25)-C(26)-C(22)$ & $109.0(3)$ \\
\hline$C(13)-C(11)-C(14)$ & $105.7(3)$ & $\mathrm{C}(25)-\mathrm{C}(26)-\mathrm{Hf}(1)$ & $71.2(2)$ \\
\hline $\mathrm{C}(12)-\mathrm{C}(11)-\mathrm{C}(14)$ & $107.1(3)$ & $C(22)-C(26)-H f(1)$ & $74.5(2)$ \\
\hline$C(13)-C(11)-S i(3)$ & $116.0(3)$ & $\mathrm{C}(22)-\mathrm{C}(27)-\mathrm{C}(28)$ & $112.3(4)$ \\
\hline$C(12)-C(11)-S i(3)$ & $108.8(3)$ & $\mathrm{C}(33)-\mathrm{C}(29)-\mathrm{C}(30)$ & $106.7(3)$ \\
\hline$C(14)-C(11)-S i(3)$ & $110.2(3)$ & $C(33)-C(29)-C(34)$ & $126.9(4)$ \\
\hline$C(16)-C(15)-C(18)$ & $107.3(3)$ & $C(30)-C(29)-C(34)$ & $125.9(4)$ \\
\hline$C(16)-C(15)-C(17)$ & $106.8(3)$ & $\mathrm{C}(33)-\mathrm{C}(29)-\mathrm{Hf}(1)$ & $72.0(2)$ \\
\hline$C(18)-C(15)-C(17)$ & $107.0(3)$ & $\mathrm{C}(30)-\mathrm{C}(29)-\mathrm{Hf}(1)$ & $72.0(2)$ \\
\hline$C(16)-C(15)-S i(3)$ & $116.2(3)$ & $C(34)-C(29)-H f(1)$ & $126.8(3)$ \\
\hline$C(18)-C(15)-\operatorname{Si}(3)$ & $108.3(3)$ & $C(31)-C(30)-C(29)$ & $109.5(4)$ \\
\hline$C(17)-C(15)-S i(3)$ & $110.8(2)$ & $C(31)-C(30)-H f(1)$ & $71.8(2)$ \\
\hline$C(20)-P(1)-C(19)$ & $100.7(2)$ & $C(29)-C(30)-H f(1)$ & $75.5(2)$ \\
\hline$C(20)-P(1)-C(21)$ & $99.92(19)$ & $\mathrm{C}(30)-\mathrm{C}(31)-\mathrm{C}(32)$ & $107.7(3)$ \\
\hline$C(19)-P(1)-C(21)$ & $100.9(2)$ & $\mathrm{C}(30)-\mathrm{C}(31)-\mathrm{Hf}(1)$ & $75.3(2)$ \\
\hline$C(20)-P(1)-H f(1)$ & $117.44(13)$ & $\mathrm{C}(32)-\mathrm{C}(31)-\mathrm{Hf}(1)$ & $73.2(2)$ \\
\hline$C(19)-P(1)-H f(1)$ & $118.77(13)$ & $\mathrm{C}(31)-\mathrm{C}(32)-\mathrm{C}(33)$ & $107.3(3)$ \\
\hline $\mathrm{C}(21)-\mathrm{P}(1)-\mathrm{Hf}(1)$ & $115.98(14)$ & $\mathrm{C}(31)-\mathrm{C}(32)-\mathrm{Hf}(1)$ & $73.4(2)$ \\
\hline
\end{tabular}




$\begin{array}{lrlr}\mathrm{C}(33)-\mathrm{C}(32)-\mathrm{Hf}(1) & 75.0(2) & \mathrm{C}(32)-\mathrm{C}(33)-\mathrm{Hf}(1) & 71.5(2) \\ \mathrm{C}(29)-\mathrm{C}(33)-\mathrm{C}(32) & 108.8(4) & \mathrm{C}(29)-\mathrm{C}(34)-\mathrm{C}(35) & 113.8(3) \\ \mathrm{C}(29)-\mathrm{C}(33)-\mathrm{Hf}(1) & 75.6(2) & & \end{array}$

Symmetry transformations used to generate equivalent atoms: 
Table 4. Anisotropic displacement parameters $\left(\AA^{2} \times 10^{3}\right)$ for $\left(\eta-\mathrm{C}_{5} \mathrm{H}_{4} \mathrm{Et}\right)_{2}\left(\mathrm{PMe}_{3}\right) \mathrm{Hf}=\mathrm{Si}\left(\mathrm{SiMe}^{t} \mathrm{Bu}_{2}\right)_{2}(6)$.

The anisotropic displacement factor exponent takes the form: $\quad-2 \pi^{2}\left[h^{2} a^{* 2} U^{11}+\ldots+2 h k a^{*} b^{*} U^{12}\right]$

\begin{tabular}{|c|c|c|c|c|c|c|}
\hline & $\mathrm{U}^{11}$ & $\mathrm{U}^{22}$ & $\mathrm{U}^{33}$ & $\mathrm{U}^{23}$ & $\mathrm{U}^{13}$ & $\mathrm{U}^{12}$ \\
\hline $\operatorname{Si}(1)$ & $22(1)$ & $18(1)$ & $26(1)$ & $0(1)$ & $12(1)$ & $1(1)$ \\
\hline $\mathrm{Hf}(1)$ & $21(1)$ & $19(1)$ & $24(1)$ & $0(1)$ & $11(1)$ & $1(1)$ \\
\hline $\operatorname{Si}(2)$ & $28(1)$ & $19(1)$ & $32(1)$ & $1(1)$ & $17(1)$ & $3(1)$ \\
\hline$C(1)$ & $35(2)$ & $25(2)$ & $40(2)$ & $1(1)$ & $23(2)$ & $4(1)$ \\
\hline$C(2)$ & $40(2)$ & $22(2)$ & $42(2)$ & $-3(1)$ & $26(2)$ & $-4(1)$ \\
\hline$C(3)$ & $54(2)$ & $26(2)$ & $52(2)$ & $1(2)$ & $35(2)$ & $-4(2)$ \\
\hline$C(4)$ & $57(3)$ & $23(2)$ & $63(3)$ & $-9(2)$ & $40(2)$ & $-2(2)$ \\
\hline$C(5)$ & $43(2)$ & $31(2)$ & $41(2)$ & $-7(2)$ & $24(2)$ & $-5(2)$ \\
\hline$C(6)$ & $29(2)$ & $29(2)$ & $37(2)$ & $7(1)$ & $17(1)$ & $8(1)$ \\
\hline$C(7)$ & $39(2)$ & $41(2)$ & $48(2)$ & $14(2)$ & $23(2)$ & $20(2)$ \\
\hline$C(8)$ & $28(2)$ & $46(2)$ & $38(2)$ & $9(2)$ & $13(2)$ & $0(2)$ \\
\hline$C(9)$ & $32(2)$ & $39(2)$ & $32(2)$ & $7(2)$ & $14(1)$ & $7(2)$ \\
\hline $\operatorname{Si}(3)$ & $25(1)$ & $19(1)$ & $27(1)$ & $0(1)$ & $14(1)$ & $-2(1)$ \\
\hline$C(10)$ & $32(2)$ & $23(2)$ & $40(2)$ & $2(1)$ & $19(2)$ & $1(1)$ \\
\hline$C(11)$ & $31(2)$ & $27(2)$ & $30(2)$ & $1(1)$ & $15(1)$ & $-3(1)$ \\
\hline$C(12)$ & $39(2)$ & $42(2)$ & $35(2)$ & $-9(2)$ & $18(2)$ & $-12(2)$ \\
\hline$C(13)$ & $41(2)$ & $38(2)$ & $33(2)$ & $-1(1)$ & $22(2)$ & $-1(2)$ \\
\hline$C(14)$ & $43(2)$ & $34(2)$ & $31(2)$ & $5(2)$ & $14(2)$ & $3(2)$ \\
\hline$C(15)$ & $24(2)$ & $30(2)$ & $32(2)$ & $1(1)$ & $15(1)$ & $-5(1)$ \\
\hline$C(16)$ & $27(2)$ & $33(2)$ & $39(2)$ & $9(2)$ & $15(2)$ & $1(1)$ \\
\hline$C(17)$ & $31(2)$ & $32(2)$ & $47(2)$ & $5(2)$ & $20(2)$ & $-4(2)$ \\
\hline $\mathrm{C}(18)$ & $36(2)$ & $43(2)$ & $38(2)$ & $-10(2)$ & $14(2)$ & $-14(2)$ \\
\hline $\mathrm{P}(1)$ & $28(1)$ & $25(1)$ & $29(1)$ & $-4(1)$ & $13(1)$ & $-2(1)$ \\
\hline$C(19)$ & $31(2)$ & $40(2)$ & $36(2)$ & $-7(2)$ & $10(2)$ & $1(2)$ \\
\hline$C(20)$ & $39(2)$ & $29(2)$ & $38(2)$ & $-4(2)$ & $19(2)$ & $-8(2)$ \\
\hline$C(21)$ & $48(2)$ & $34(2)$ & $33(2)$ & $-8(2)$ & $23(2)$ & $-3(2)$ \\
\hline$C(22)$ & $32(2)$ & $28(2)$ & $32(2)$ & $-2(1)$ & $18(1)$ & $-3(1)$ \\
\hline$C(23)$ & $27(2)$ & $31(2)$ & $33(2)$ & $0(1)$ & $16(1)$ & $-6(1)$ \\
\hline$C(24)$ & $35(2)$ & $24(2)$ & $39(2)$ & $-3(1)$ & $23(2)$ & $-6(2)$ \\
\hline$C(25)$ & $29(2)$ & $25(2)$ & $40(2)$ & $5(1)$ & $19(2)$ & $3(1)$ \\
\hline$C(26)$ & $29(2)$ & $27(2)$ & $32(2)$ & $1(1)$ & $14(1)$ & $-4(1)$ \\
\hline
\end{tabular}




\begin{tabular}{lcccccc}
$\mathrm{C}(27)$ & $39(2)$ & $42(2)$ & $42(2)$ & $0(2)$ & $27(2)$ & $-1(2)$ \\
$\mathrm{C}(28)$ & $106(5)$ & $67(4)$ & $184(8)$ & $-33(4)$ & $127(6)$ & $-26(4)$ \\
$\mathrm{C}(29)$ & $25(2)$ & $31(2)$ & $36(2)$ & $5(1)$ & $13(1)$ & $4(1)$ \\
$\mathrm{C}(30)$ & $29(2)$ & $36(2)$ & $39(2)$ & $6(2)$ & $19(2)$ & $9(2)$ \\
$\mathrm{C}(31)$ & $36(2)$ & $25(2)$ & $36(2)$ & $2(1)$ & $19(2)$ & $12(2)$ \\
$\mathrm{C}(32)$ & $33(2)$ & $24(2)$ & $37(2)$ & $2(1)$ & $16(2)$ & $5(1)$ \\
$\mathrm{C}(33)$ & $28(2)$ & $28(2)$ & $28(2)$ & $2(1)$ & $11(1)$ & $4(1)$ \\
$\mathrm{C}(34)$ & $25(2)$ & $41(2)$ & $35(2)$ & $4(2)$ & $9(2)$ & $-1(2)$ \\
$\mathrm{C}(35)$ & $33(2)$ & $46(2)$ & $37(2)$ & $-5(2)$ & $10(2)$ & $-9(2)$ \\
& & & & & & \\
\hline
\end{tabular}


Table 5. Hydrogen coordinates $\left(\times 10^{4}\right)$ and isotropic displacement parameters $\left(\AA^{2} \times 10^{3}\right)$ for $\left(\eta-\mathrm{C}_{5} \mathrm{H}_{4} \mathrm{Et}\right)_{2}\left(\mathrm{PMe}_{3}\right)-\mathrm{Hf}=\mathrm{Si}\left(\mathrm{SiMe}^{\prime} \mathrm{Bu}_{2}\right)_{2}(6)$.

\begin{tabular}{|c|c|c|c|c|}
\hline & $x$ & $y$ & $\mathrm{z}$ & $\mathrm{U}(\mathrm{eq})$ \\
\hline $\mathrm{H}(1)$ & 5935 & 3335 & 7575 & 47 \\
\hline $\mathrm{H}(2)$ & 6743 & 2765 & 8204 & 47 \\
\hline $\mathrm{H}(3)$ & 6036 & 1983 & 7603 & 47 \\
\hline $\mathrm{H}(4)$ & 7061 & 5078 & 6333 & 60 \\
\hline $\mathrm{H}(5)$ & 7840 & 4319 & 6590 & 60 \\
\hline $\mathrm{H}(6)$ & 7891 & 5494 & 7021 & 60 \\
\hline $\mathrm{H}(7)$ & 7322 & 5706 & 7893 & 64 \\
\hline $\mathrm{H}(8)$ & 6808 & 4712 & 7996 & 64 \\
\hline $\mathrm{H}(9)$ & 6482 & 5362 & 7188 & 64 \\
\hline $\mathrm{H}(10)$ & 8480 & 4566 & 8292 & 55 \\
\hline$H(11)$ & 8455 & 3336 & 7933 & 55 \\
\hline $\mathrm{H}(12)$ & 8046 & 3534 & 8471 & 55 \\
\hline $\mathrm{H}(13)$ & 5687 & 4824 & 6008 & 63 \\
\hline $\mathrm{H}(14)$ & 5233 & 4144 & 6387 & 63 \\
\hline $\mathrm{H}(15)$ & 4865 & 4201 & 5469 & 63 \\
\hline $\mathrm{H}(16)$ & 4783 & 2157 & 5288 & 57 \\
\hline $\mathrm{H}(17)$ & 5043 & 2061 & 6188 & 57 \\
\hline $\mathrm{H}(18)$ & 5510 & 1357 & 5832 & 57 \\
\hline $\mathrm{H}(19)$ & 6228 & 2421 & 5247 & 52 \\
\hline $\mathrm{H}(20)$ & 6400 & 3752 & 5406 & 52 \\
\hline $\mathrm{H}(21)$ & 5525 & 3320 & 4823 & 52 \\
\hline $\mathrm{H}(22)$ & 7500 & -2293 & 8149 & 47 \\
\hline $\mathrm{H}(23)$ & 8205 & -1592 & 8123 & 47 \\
\hline $\mathrm{H}(24)$ & 7460 & -1993 & 7337 & 47 \\
\hline $\mathrm{H}(25)$ & 8022 & 1588 & 8874 & 58 \\
\hline $\mathrm{H}(26)$ & 8614 & 537 & 9056 & 58 \\
\hline $\mathrm{H}(27)$ & 8442 & 1039 & 9719 & 58 \\
\hline $\mathrm{H}(28)$ & 7178 & 743 & 9701 & 53 \\
\hline $\mathrm{H}(29)$ & 6482 & 22 & 9034 & 53 \\
\hline $\mathrm{H}(30)$ & 6692 & 1265 & 8852 & 53 \\
\hline
\end{tabular}




\begin{tabular}{|c|c|c|c|c|}
\hline $\mathrm{H}(31)$ & 8259 & -1359 & 9348 & 55 \\
\hline $\mathrm{H}(32)$ & 7406 & -1581 & 9289 & 55 \\
\hline $\mathrm{H}(33)$ & 8047 & -822 & 9976 & 55 \\
\hline $\mathrm{H}(34)$ & 5358 & 596 & 6959 & \\
\hline $\mathrm{H}(35)$ & 5497 & 268 & 7804 & \\
\hline $\mathrm{H}(36)$ & 4848 & -401 & 7067 & 30 \\
\hline $\mathrm{H}(37)$ & 6070 & -1658 & 8362 & 54 \\
\hline $\mathrm{H}(38)$ & 6305 & -2514 & 7871 & 54 \\
\hline $\mathrm{H}(39)$ & 5393 & -2209 & 7586 & 54 \\
\hline $\mathrm{H}(40)$ & 5217 & -1599 & 6309 & 50 \\
\hline $\mathrm{H}(41)$ & 6115 & -1985 & 6592 & 60 \\
\hline $\mathrm{H}(42)$ & 5832 & -737 & 6248 & 60 \\
\hline $\mathrm{H}(43)$ & 6152 & -381 & 4367 & 57 \\
\hline $\mathrm{H}(44)$ & 6550 & 853 & 4571 & 57 \\
\hline $\mathrm{H}(45)$ & 6214 & 292 & 5101 & 57 \\
\hline $\mathrm{H}(46)$ & 6910 & -1835 & 5906 & 53 \\
\hline $\mathrm{H}(47)$ & 7617 & -2407 & 5807 & 53 \\
\hline $\mathrm{H}(48)$ & 6763 & -2243 & 5072 & 53 \\
\hline $\mathrm{H}(49)$ & 7324 & -1298 & 4238 & 55 \\
\hline $\mathrm{H}(50)$ & 8230 & -1365 & 4895 & 55 \\
\hline $\mathrm{H}(51)$ & 7873 & -189 & 4460 & 55 \\
\hline $\mathrm{H}(52)$ & 9924 & 2140 & 6923 & 36 \\
\hline $\mathrm{H}(53)$ & 8833 & 3079 & 7097 & 37 \\
\hline $\mathrm{H}(54)$ & 7533 & 2728 & 5887 & 36 \\
\hline $\mathrm{H}(55)$ & 7838 & 1628 & 4957 & 35 \\
\hline $\mathrm{H}(56)$ & 9128 & 1049 & 4871 & 45 \\
\hline $\mathrm{H}(57)$ & 9659 & 341 & 5641 & 4 \\
\hline $\mathrm{H}(58)$ & 10083 & 2611 & 5531 & 142 \\
\hline $\mathrm{H}(59)$ & 10355 & 1609 & 5157 & 142 \\
\hline $\mathrm{H}(60)$ & 10649 & 1621 & 6066 & 142 \\
\hline $\mathrm{H}(61)$ & 10194 & -59 & 6922 & 4 \\
\hline $\mathrm{H}(62)$ & 9127 & -1525 & 6246 & 38 \\
\hline $\mathrm{H}(63)$ & 8402 & -1806 & 7036 & 38 \\
\hline $\mathrm{H}(64)$ & 9042 & -491 & 8200 & 34 \\
\hline $\mathrm{H}(65)$ & 10543 & 1440 & 8126 & 4. \\
\hline $\mathrm{H}(66)$ & 10906 & 378 & 8693 & \\
\hline
\end{tabular}


$\mathrm{H}(67)$

9645

1763

8618

62

$\mathrm{H}(68)$

10541

1809

9298

62

$\mathrm{H}(69)$

10011

702

9188

62 